\title{
Equilibrium and Nonequilibrium States in Microfluidic Double Emulsions
}

Pannacci, N.; Bruus, Henrik; Bartolo, D.; Etchart, I.; Lockhart, T.; Hennequin, Y.; Willaime, H.; Tabeling, P.

Published in:

Physical Review Letters

Link to article, DOI:

10.1103/PhysRevLett.101.164502

Publication date:

2008

Document Version

Publisher's PDF, also known as Version of record

Link back to DTU Orbit

Citation (APA):

Pannacci, N., Bruus, H., Bartolo, D., Etchart, I., Lockhart, T., Hennequin, Y., Willaime, H., \& Tabeling, P. (2008). Equilibrium and Nonequilibrium States in Microfluidic Double Emulsions. Physical Review Letters, 101(16), 164502. https://doi.org/10.1103/PhysRevLett.101.164502

\section{General rights}

Copyright and moral rights for the publications made accessible in the public portal are retained by the authors and/or other copyright owners and it is a condition of accessing publications that users recognise and abide by the legal requirements associated with these rights.

- Users may download and print one copy of any publication from the public portal for the purpose of private study or research.

- You may not further distribute the material or use it for any profit-making activity or commercial gain

- You may freely distribute the URL identifying the publication in the public portal 


\title{
Equilibrium and Nonequilibrium States in Microfluidic Double Emulsions
}

\author{
Nicolas Pannacci, ${ }^{1}$ Henrik Bruus, ${ }^{2}$ Denis Bartolo, ${ }^{1}$ Isabelle Etchart, ${ }^{1}$ Thibaut Lockhart, ${ }^{1}$ Yves Hennequin, ${ }^{1}$ \\ Hervé Willaime, ${ }^{1}$ and Patrick Tabeling ${ }^{1}$ \\ ${ }^{1}$ Laboratoire MMN, CNRS-ESPCI, 10 Rue Vauquelin, 75005 Paris, France \\ ${ }^{2}$ Department of Micro- and Nanotechnology, Technical University of Denmark DTU Nanotech, \\ Building 345 East, DK-2800 Kongens Lyngby, Denmark
}

(Received 15 January 2008; revised manuscript received 30 June 2008; published 14 October 2008; publisher error corrected 15 October 2008)

\begin{abstract}
We describe experimental and theoretical studies dedicated to establishing the physics of formation of double droplets in microfluidic systems. We show that the morphologies (complete engulfing, partial engulfing, and nonengulfing) obtained at late times minimize the interfacial energy of the system. We explain that nonequilibrium morphologies generated in the system can have long lifetimes. Remarkably, the physics of formation of the double droplets with microfluidics allows the synthesis of particles with new morphologies.
\end{abstract}

Double emulsions (i.e., emulsions in which the dispersed phase is also an emulsion) are of considerable interest in food, cosmetic industries, medical sciences (drug delivery), printing, and textile industries [1]. Traditional techniques of production of double emulsions essentially consist in mixing three fluids together. Owing to a lack of control of the kinetics of the formation of the structures, one typically obtains emulsions with broad size distributions and polymorphism. Recently, a number of investigators succeeded in generating double emulsions in microfluidic systems [2-4] and in capillaries [5]. The experiments generated remarkably monodisperse structures in which the number of droplets inside each globule could be controlled [2-5]. However stimulating the results can be, the type of structure which can be obtained in a given microfluidic geometry, at different stages of their formation, and for prescribed triplets of liquids remains unclear. Phase diagrams published recently in the literature [3] suggest that different morphologies can be obtained with the same fluids, which is puzzling from the standpoint of mechanical equilibrium. What we show in the present Letter is that one may obtain on the same chip equilibrium and nonequilibrium structures (that may correspond to different morphologies), depending on the range of time we address. Owing to the excellent control of the kinetics of formation, nonequilibrium states can be photocured to elaborate new particles well beyond the possibilities of traditional technologies.

The microsystems are made in polydimethylsiloxane (PDMS), using soft lithography technique [6,7]. The systems have a double flow focusing geometry similar to Ref. [3,8] where two junctions are placed in series (see Fig. 1). In such a geometry, an emulsion of $A / B$ is produced at the first junction. The structures travel downstream in a second junction, where eventually, the double emulsions $A / B / C$ are formed. The structures are further stored in a reservoir. The microchannels have rectangular cross sections, typically $100 \mu \mathrm{m}$ deep and $200 \mu \mathrm{m}$ wide, with a length of $2 \mathrm{~cm}$ for the main channel. In most cases, the microsystems are sealed after a $30 \mathrm{~s}$ exposure in an oxygen plasma cleaner. The resulting hydrophilicity of the PDMS microchannel surfaces $[9,10]$ allows generation of $(O / O / W)$ double emulsions. In other cases the PDMS walls of the channels are hydrophobic (after plasma sealing, hydrophobic recovery is accelerated in an oven) and $(\mathrm{W} / \mathrm{O} / \mathrm{O})$ double emulsions are generated.

The working fluids are alkanes, octanol, rapeseed oil, fluorinated oil, mineral oil, silicone oil, tripropylene glycol diacrylate (TPGDA), and water without and with sodium dodecyl sulfate (SDS) surfactant $(1 \% \mathrm{w} / \mathrm{w})$. Chemical products are commercial, with a purity of $99 \%$. The inter-

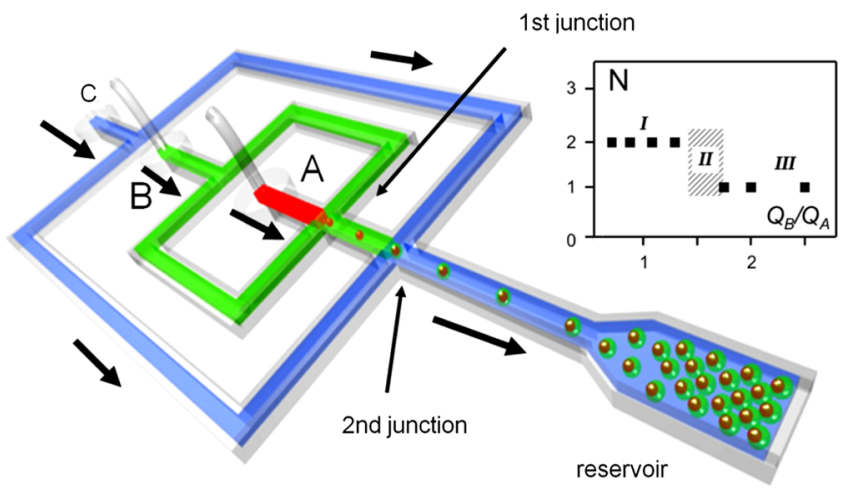

FIG. 1 (color online). Double flow focusing geometry used in the experiment: a first structure $A / B$ is produced at the first junction. Downstream fluid $C$ engulfs the structure, and a structure $A / B / C$ is formed. The structure further moves into a reservoir. In the upper right figure, $N$ is the number of droplets encapsulated in a tetradecane/TPGDA/water $+1 \%$ SDS system, and $Q_{A}$ and $Q_{B}$ are, respectively, the flow rates of fluids $A$ and $B$. I and III are monodisperse regimes and II (shaded region) represents polydisperse regimes for which the emulsion includes globules engulfing one or two droplets. 
facial tensions corresponding to the three fluid interfaces are measured by using pendant drop method or ring technique. We systematically mix the three fluids together before performing the measurements so as equilibrium is reached, both from the viewpoints of surface energies and chemical potentials (solubilities).

To produce double emulsions, we drive the three fluids into the microsystem, tuning pressure or flow rates so as the droplets are formed sequentially at the first and second junctions. Typical orders of magnitude are $10 \mu \mathrm{L} \min ^{-1}$ for the flow rates and between 100 mbar and 1 bar for the inlet pressures. Throughout the experiments, Reynolds and capillary numbers are small.

The observations we report here are obtained by working with ratios of emission frequencies at the two junctions equal to unity. In such situations, the structures produced in the system have unique morphologies with no repeated pattern. When the ratio of the emission frequencies is not equal to unity, the system produces repeated patterns, such as several identical droplets engulfed in each globule. There also exist regimes where the system generates nonunique structures, such as globules engulfing different numbers of droplets. The domains of existence of the various regimes are illustrated in the inset of Fig. 1. The plot reveals the existence of plateaus where the ratios of the emission frequencies are locked. These resonance plateaus, which evoke devil's staircases, result from the fact that in the second junction, droplets arriving at fixed frequencies perturb the flow, giving rise to resonance phenomena similar to those described in Ref. [11]. Such phenomena favor the monodispersivity of the structures the system generates, making feasible the formation of perfect crystals and are therefore worth being pointed out.

We now analyze the "late time morphologies" of the structures we produced. These morphologies are studied in the reservoir a few minutes after the flow is interrupted. We checked that such morphologies do not evolve for hours and are unique for given triplets of fluids, independently of the flow conditions. Varying the flow rates (however keeping the emission frequencies ratio equal to unity) changes sizes but not morphologies. Figure 2 shows two wellordered arrays of late time structures. Two morphologies are shown: partially engulfed (or Janus) and completely engulfed. The third morphology that we obtained at late times is nonengulfing, i.e., droplets remaining separated. The ensemble of observations we made for 30 triplets of fluids are summarized in Fig. 3, using spreading parameters as coordinates in a way similar to Refs. [12,13]: $S_{i}=$ $\gamma_{j k}-\left(\gamma_{i j}+\gamma_{i k}\right)$, where $\gamma_{i j}$ are the interfacial tensions between fluid $i$ and $j$. We also represent the morphologies obtained by minimizing the interfacial energy of the system. In this diagram, there exist mathematically forbidden regions. We also represent a region which, according to the Girifalco relation [14], is forbidden. Figure 3 shows that the late time structures appear to minimize the interfacial

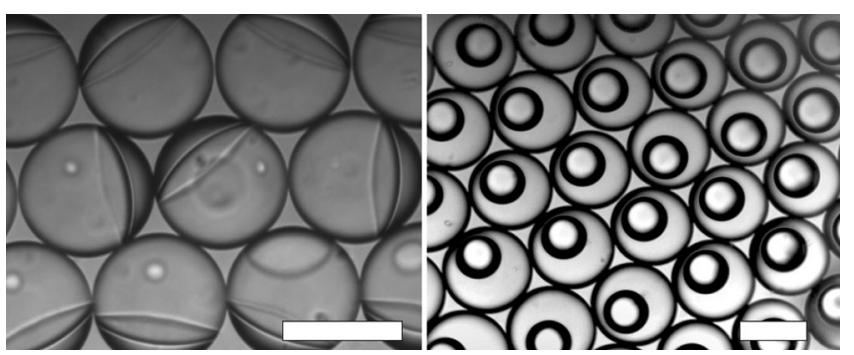

FIG. 2. Two late time morphologies obtained in the experiments. On the left, partial engulfing (or Janus state) obtained using silicone oil and rapeseed oil, with distilled water as the external phase. On the right, complete engulfing obtained using fluorinated oil/silicone oil and water + SDS as the external phase. The white bars represent $100 \mu \mathrm{m}$.

energy of the system. This is clear when the spreading coefficients $S_{2}$ and $S_{3}$ are well above the experimental uncertainty, i.e., $\pm 1 \mathrm{mN} / \mathrm{m}$. Agreement between theory and experiment is more questionable at low values of $S_{3}$. Systematically, Janus seem located slightly above the horizontal axis. Nonetheless, such a discrepancy is close to the experimental uncertainty. Thus one may conclude that as a whole, the diagram of Fig. 3 supports the idea that the late time structures obtained in our microfluidic experiment correspond to mechanical equilibria.

We now turn our attention to the early time morphologies, i.e., those traveling from the second junction where they are formed towards the reservoir. Two examples of such structures are shown in Fig. 4. In the first case [Fig. 4(a)], complete engulfment is observed, while the corresponding equilibrium morphology is nonengulfing. The second case [Fig. 4(b)] also shows complete engulfing at short time, while Janus is the equilibrium state. In fact, the dynamics towards equilibrium can be classified into

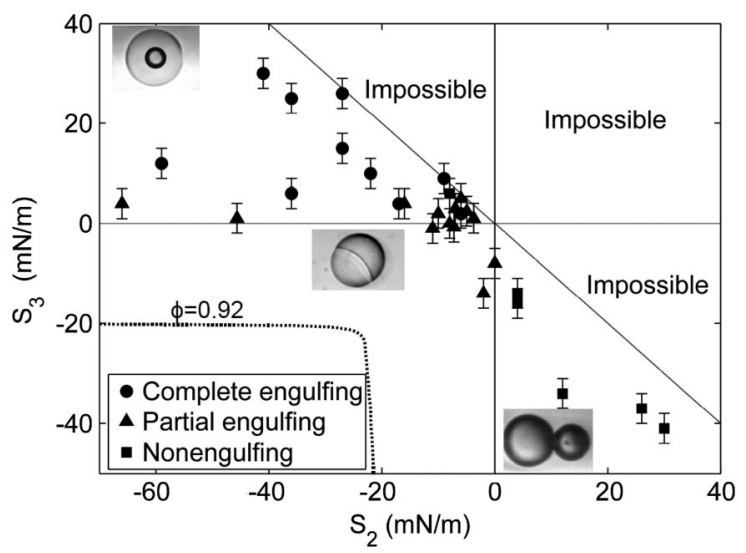

FIG. 3. Comparison between equilibrium and observed morphologies: Squares represent nonengulfing, circles engulfing, and triangles partial engulfing, as observed experimentally. The dotted line defines the boundary of a physically inaccessible region, as suggested by Girifalco's formula, with the $\phi$ parameter (cf. Ref. [13]) equal to 0.92 . 

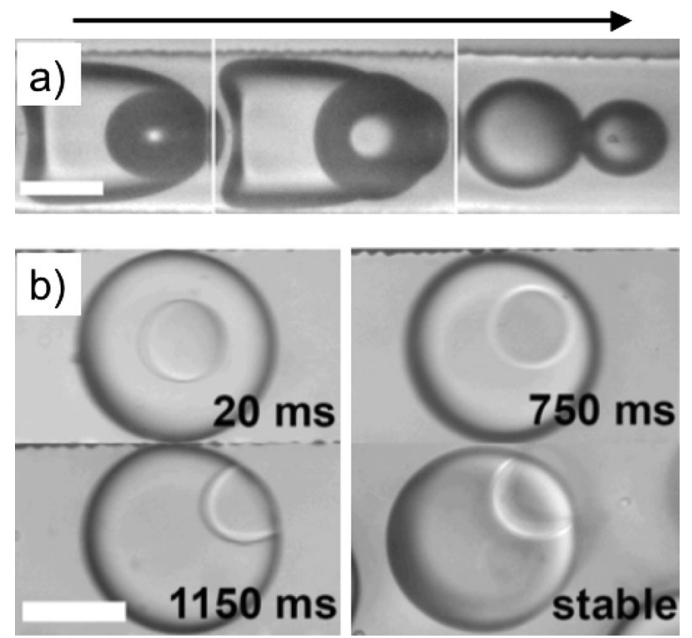

FIG. 4. (a) Evolution from an encapsulated to a nonencapsulated state; using fluorinated oil/water + SDS/TPGDA. The entire process takes less than $1 \mathrm{~ms}$. The arrow indicates the flow direction. (b) Evolution from a complete encapsulation to a Janus state, which represents the equilibrium in this case; the fluids are tetradecane/TPGDA/water $+1 \% \mathrm{w} / \mathrm{w}$ SDS. White bars represent $100 \mu \mathrm{m}$.

several types, depending on whether a contact line common to the three fluids appears during their formation. The transient regime also depends on the nature of the equilibrium state towards which the system evolves. We examine two possibilities here. In Fig. 4(a), the three fluids turn out to develop a common line during the formation process. In this case, the system evolves almost immediately towards a structure close to the equilibrium state. The time it takes to reach equilibrium in such a case is less than $1 \mathrm{~ms}$, which is comparable to a capillary time [15]. In the second example shown in Fig. 4(b), one phase remains physically separated from the other during the formation process (i.e., a small droplet inside a large host droplet). In such a case, the evolution towards equilibrium is much slower, taking hundreds of milliseconds or so. In fact, the inner droplet must touch the inner boundary of its host to evolve to equilibrium under the action of capillary forces. We analyzed in some detail this process by measuring the temporal evolution of the distance $d$ between the center of the inner droplet and the interface of the host droplet, the origin of time being taken as the double droplet is formed at the second junction. In Fig. 5, the reduced distance $d / a, a$ being the radius of the host droplet, is plotted versus the reduced time $t / \tau$ (where $\tau$ is the time it takes for the inner droplet to touch the interface of the host droplet) for three different experiments. We checked the trajectories of the inner droplet lies along a diameter, parallel to the main stream. In the three cases, the dynamics of the process is roughly linear in time, with $\tau$ ranging between 0.7 and $1.3 \mathrm{~s}$.

We present here a model dedicated to estimate the lifetimes of nonequilibrium engulfing morphologies in situ-

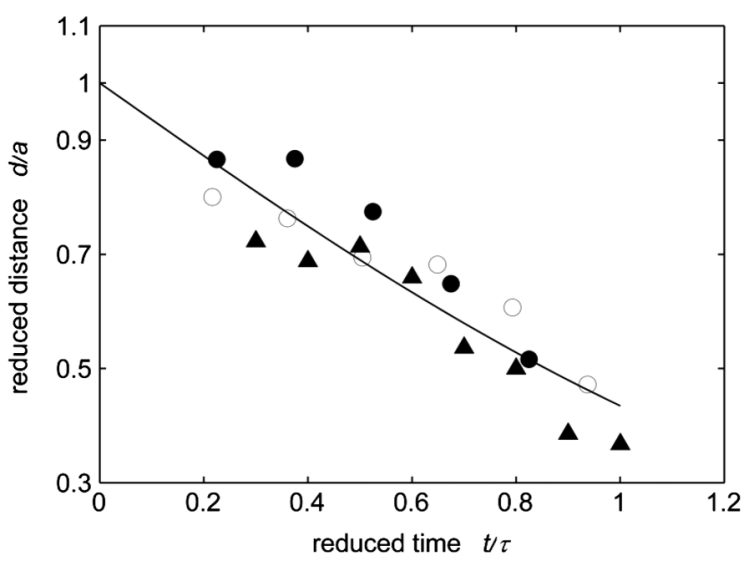

FIG. 5. Reduced distance $d / a$ from the center of the inner droplet to the interface of the host drop versus reduced time $t / \tau$ in the transient regime. Open circles, circles, and triangles are obtained, respectively, in the following conditions: (a) $a=65 \mu \mathrm{m}, 98 \mu \mathrm{m}, 92 \mu \mathrm{m}$; (b) $\hat{a}=26 \mu \mathrm{m}, 37 \mu \mathrm{m}$, $40 \mu \mathrm{m} ; \quad$ (c) $\lambda=0.4, \quad 0.38, \quad 0.43 ; U=$ (a) $20.4 \mathrm{~mm} / \mathrm{s}$, (b) $10.3 \mathrm{~mm} / \mathrm{s}$, (c) $9.3 \mathrm{~mm} / \mathrm{s}$. For experiments a, b, and c, the viscosity of the inner droplet is $\mu_{0}=3 \mathrm{mPas}$, that of the host drop is $\hat{\mu}=15 \mathrm{mPas}$; the viscosity of the continuous phase is $\mu=1 \mathrm{mPa}$ s for experiments a and $\mathrm{b}$ and $2.4 \mathrm{mPa}$ s for experiment $\mathrm{c}$ due to addition of glycerol. The typical lifetimes or the transient regimes are $\tau=$ (a) $0.68 \mathrm{~s}$, (b) $0.78 \mathrm{~s}$, and (c) $1.29 \mathrm{~s}$ while the predicted times are, respectively, $0.1 \mathrm{~s}, 0.2 \mathrm{~s}$, and $0.1 \mathrm{~s}$. The line shows the theoretical prediction developed in the paper.

ations where the equilibrium state is a Janus. The model is based on the idea that the inner droplet is displaced under the action of internal recirculations in the host drop. In the model, the droplet behaves as a small neutrally buoyant particle advected by the internal flow. We use here Hadamard-Rybzynski analysis [16-19] for a drop of radius $a$, of viscosity $\hat{\mu}$ moving with steady velocity $U$ along the $x$ axis inside a surrounding liquid of viscosity $\mu$. The representation of the internal circulation is consistent with the experimental and numerical work of Ref. [20]. Choosing a coordinate system with its origin at the steadily moving center-of-mass of the drop, and introducing the viscosity ratio $\lambda=\frac{\hat{\mu}}{\mu}$, the relative velocity $V$ of the droplet is given by

$$
V=\frac{d x}{d t}=\frac{1}{2(1+\lambda)}\left(1-\frac{x^{2}}{a^{2}}\right) U .
$$

We define the displacement time $\tau_{\text {th }}$ as the time it takes the inner droplet of radius $\hat{a}$ to move along the center axis from the center $x=0$ to the interface $x=a-\hat{a}$ of the host drop. Using Eq. (1) we obtain

$$
\tau_{\mathrm{th}} \approx \int_{0}^{a-\hat{a}} \frac{d x}{V}=(1+\lambda) \ln \left(\frac{2-\alpha}{\alpha}\right) \frac{a}{U} .
$$

A typical example of the evolution of $x(t)$ with time is shown in Fig. 5. The figure indicates consistency between theory and experiment. 

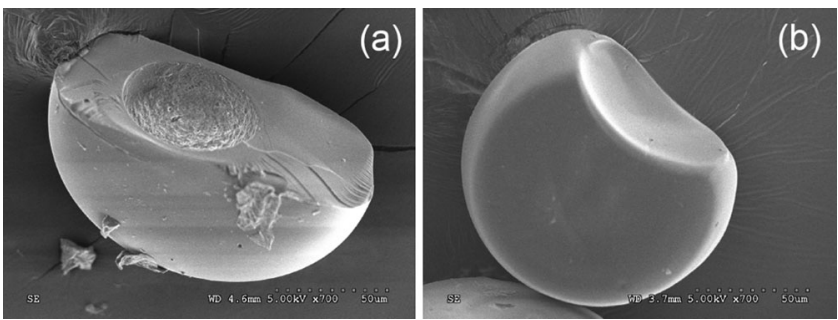

FIG. 6. SEM pictures of two different morphologies obtained with the same fluids, in the same chip, and cured under UV light: (a) nonequilibrium structure (cut with a razor blade so as to reveal full encapsulation), (b) equilibrium structure in the form of Janus particle. The fluids are tetradecane, acrylate monomer $+8 \%$ Darocur, and water $+1 \%$ SDS.

When inserting the experimental values for $\lambda, \alpha, a$, and $U$ in Eq. (2) we obtain displacement times $\tau_{\text {th }}$ ranging between 0.1 and 0.2 s, i.e., smaller than in the experiment. This is probably due to the fact that the microchannel walls and the perturbation of the internal circulation caused by the host droplet are neglected in the theory. Nonetheless, the theoretical model roughly captures an order of magnitude for the lifetime of the nonequilibrium structure and thus may be used as a starting point.

In practice, the lifetime of the nonequilibrium engulfed state allows production of new particles. This is shown in Fig. 6 in which we used a photocurable polymer as one of the three working fluids. We could photocure this monomer in less than $100 \mathrm{~ms}$, using a technique similar to [21]. Figure 6 shows that the same system, with the same flow conditions, generates fully encapsulated particles at early times [Fig. 6(a)] and Janus particles at longer times [Fig. 6(b)]. This illustrates the advantage one can draw out from the excellent control of the kinetics of formation offered by microfluidic technology.

In conclusion, we show that two families of morphologies of double droplets can be obtained under control with microfluidics: equilibrium structures, and nonequilibrium structures with lifetimes controlled by the hydrodynamics. Microfluidics allows generation of entirely monodisperse structures (owing to a resonance phenomenon), which can be assembled to form crystals or photocured to form new particles, or both, opening routes towards the elaboration of new materials.

We thank D. Barthès-Biesel, D. Bonn, D. Weitz, H. Stone, E. Kumacheva, E. Raphaël, A. Ajdari,
Y. Pomeau, E. Lorenceau, M. Schindler, and S. Pasquali for fruitful discussions. Thanks to J. Bico and to J. Mortreux for their help for interfacial tension measurements and to F. Monti for SEM pictures. We acknowledge CNRS, ESPCI, and Région Île-de-France for their financial support to this work.

[1] F. Leal-Calderon, V. Schmitt, and J. Bibette, Emulsion Science: Basic Principles (Springer, New York, 2007).

[2] S. Okushima, T. Nisisako, T. Torii, and T. Higuchi, Langmuir 20, 9905 (2004).

[3] Z. Nie, S. Xu, M. Seo, P.C. Lewis, and E. Kumacheva, J. Am. Chem. Soc. 127, 8058 (2005).

[4] V. Barbier, M. Tatoulian, H. Li, F. Arefi-Khonsari, A. Ajdari, and P. Tabeling, Langmuir 22, 5230 (2006).

[5] A. S. Utada, E. Lorenceau, D. R. Link, P. D. Kaplan, H. A. Stone, and D. A. Weitz, Science 308, 537 (2005).

[6] J. C. McDonald and G. M. Whitesides, Acc. Chem. Res. 35, 491 (2002).

[7] P. Tabeling, Introduction to Microfluidics (Oxford University Press, Oxford, 2005).

[8] M. Seo, C. Paquet, Z. Nie, S. Xu, and E. Kumacheva, Soft Matter 3, 986 (2007).

[9] B. T. Ginn and O. Steinbock, Langmuir 19, 8117 (2003).

[10] R. Dreyfus, H. Willaime, and P. Tabeling, Phys. Rev. Lett. 90, 144505 (2003).

[11] H. Willaime, V. Barbier, L. Kloul, S. Maine, and P. Tabeling, Phys. Rev. Lett. 96, 054501 (2006).

[12] S. Torza and S. G. Mason, Science 163, 813 (1969).

[13] S. Torza and S. G. Mason, J. Colloid Interface Sci. 33, 67 (1970).

[14] L. A. Girifalco and R. J. Good, J. Phys. Chem. 61, 904 (1957).

[15] D. Quéré, P.G. de Gennes, and F. Brochard-Wyart, Gouttes, Bulles, Perles et ondes (Belin, Paris, 2002).

[16] J. Hadamard, C. R. Acad. Sci. Ser. Gen. 152, 1735 (1911).

[17] W. Rybzynski, Bull. Int. Acad. Sci. Cracovie A 40 (1911).

[18] G. K. Batchelor, An Introduction to Fluid Dynamics (Cambridge University Press, Cambridge, England, 2000).

[19] H. A. Stone, A. Nadim, and S. H. Strogatz, J. Fluid Mech. 232, 629 (1991).

[20] F. Sarrazin, K. Loubiere, L. Prat, C. Gourdon, T. Bonometti, and J. Magnaudet, AIChE J. 52, 4061 (2006).

[21] D. Dendukuri, K. Tsoi, T. A. Hatton, and P. S. Doyle, Langmuir 21, 2113 (2005). 